\title{
An Effective Simulation of Heston Model: Combining Quadratic Exponential and Exact Simulation Schemes
}

\author{
Y.F. Sun, G.Y. Zhang \\ 1School of Management \\ Guangdong University of Technology \\ Guangzhou, China
}

\author{
S.Q. Li \\ Institute of System Engineering \\ South China University of Technology \\ Guangzhou, China
}

\begin{abstract}
A new discretization scheme ES-QE by combining the exact simulation (ES) and quadratic exponential (QE) scheme is proposed to simulate the volatility process and price process of the Heston model. Performances of new scheme are investigated via European option valuation with Monte-Carlo method. Numerical results show that the ES-QE scheme has a convergent mean-squared-root error with its operating accuracy higher than QE schemes and the efficiency much higher than ES'.
\end{abstract}

Keywords-Heston model; monte-carlo; quadratic exponential; exact simulation; feller condition

\section{INTRODUCTION}

The well-known Heston model [1] , one of extensions to the celebrated Black-Scholes model [2] , has been widely-used in derivatives pricing due to its good properties such as allowance of "volatility smile" and tractability[3] , etc. However, the majority of derivatives, especially the exotic options, can't always be priced in closed-forms because of the complexity of underlying assets models [4] [5] .

Recently, more and more attention has been paid to the nearly-exact discretization to stochastic volatility models [6] $\sim[10]$. The existing schemes can be coarsely grouped into two categories. The first line is Taylor-based scheme which include the implicit Milstein scheme [6] , Euler full truncated scheme[7] , transformed volatility scheme Zhu[8] , etc. The other line is called (almost) exact scheme which consists of exact simulation [9] (ES), quadratic exponential (QE), truncated Gaussian scheme[10] (TG) and so on.

In this paper, we follow the second line and propose an ES-QE scheme by applying the ES scheme to simulate the volatility process and using the QE scheme to simulate the price process of Heston model.

II. ES-QE DISCRETIZATION SCHEME FOR HESTON MODEL

The Heston model writes

$$
\left\{\begin{array}{l}
d \ln S(t)=\left(r-\frac{1}{2} V(t)\right) d t+\sqrt{V(t)} d W_{s}(t) \\
d V(t)=\kappa(\theta-V(t)) d t+\varepsilon \sqrt{V(t)} d W_{V}(t)
\end{array}\right.
$$

with $d W_{v}(t) \cdot d W_{s}(t)=\rho d t(|\rho| \leq 1)$. By integrating Eq.(1), we have

$$
\begin{aligned}
\ln \hat{S}(t+\Delta)= & \ln \hat{S}(t)+(r-\rho \kappa \theta / \varepsilon) \Delta+(\kappa \rho / \varepsilon-1 / 2) \int_{t}^{t+\Delta} \hat{V}(u) d u+\rho / \varepsilon(\hat{V}(t+\Delta)-\hat{V}(t))+ \\
& \sqrt{1-\rho^{2}} \int_{t}^{t+\Delta} \sqrt{\hat{V}(u) d W(u)}
\end{aligned}
$$

In this paper, we design ES-QE scheme to simulate the Heston model, by citing Andersen's work about dealing with the price process (including $\int_{t}^{t+\Delta} \hat{V}(u) d u$ and $\int_{t}^{t+\Delta} \sqrt{\hat{V}(u)} d W(u)$ ) and applying the exact scheme to generate samples of $\hat{V}(t+\Delta)$ directly, instead of moment-matching as described in QE scheme.

\section{A. Simulating Volatility Process by Applying ES Scheme}

According to Cox et al[11], given $\hat{V}(t), \hat{V}(t+\Delta)$ is $\left(\varepsilon^{2}\left(1-e^{-\mu}\right) / 4 \kappa\right)$ times a non-central chi-square distributed random variable which has the freedom degree of $d=\left(4 \kappa \theta / \varepsilon^{2}\right)$ and the non-central parameter $\lambda=\left(4 \kappa e^{-\kappa \Delta} / \varepsilon^{2}\left(1-e^{-\kappa \Delta}\right)\right) \hat{V}(t)$, i.e.,

$$
\hat{V}(t+\Delta)=\left(\varepsilon^{2}\left(1-e^{-\kappa \Delta}\right) / 4 \kappa\right) \chi_{d}^{\prime 2}\left(\left(4 \kappa e^{-\kappa \Delta} / \varepsilon^{2}\left(1-e^{-\kappa \Delta}\right)\right) \hat{V}(t)\right) .
$$

We obtain the exact sampling of $\chi_{d}^{\prime 2}(\lambda)$ by referring to Johnson et. al.[12] :

a) If $d>1, \chi_{d}^{\prime 2}(\lambda)=\chi_{1}^{\prime 2}(\lambda)+\chi_{d-1}^{2}$, where $\chi_{d-1}^{2}$ is a central chi-square distributed random variable with freedom degree $d-1 \quad, \quad \chi_{1}^{\prime 2}=\left(Z_{1}+\sqrt{\lambda}\right)^{2} \quad$ and $\quad Z_{1} \sim N(0,1)$. Hence $\chi_{d}^{\prime 2}(\lambda)=\left(Z_{1}+\sqrt{\lambda}\right)^{2}+\chi_{d-1}^{2}$.

b) For any $d>0, \chi_{d}^{\prime 2}(\lambda)=\chi_{d+2 N}^{2}$, where $N$ is a Poisson random variable with mean $(1 / 2) \lambda$.

\section{B. Sampling Price Process by using QE Scheme}

Eq. (2) shows that we should have $\hat{S}(t), \hat{V}(t), \int_{t}^{t+\Delta} \hat{V}(u) d u$ and $\int_{t}^{t+\Delta} \sqrt{\hat{V}(u)} d W(u)$ in advance before sampling $\hat{S}(t+\Delta)$. 
Dufresne[13] proposed a moment function scheme to sample $\int_{t}^{t+\Delta} \hat{V}(u) d u$, i.e.,

$$
\int_{t}^{t+\Delta} \hat{V}(u) d u \approx \Delta\left[\gamma_{1} \hat{V}(t)+\gamma_{2} \hat{V}(t+\Delta)\right],
$$

where $\gamma_{1}, \gamma_{2}$ are certain constants. In this paper, we choose $\gamma_{1}=1 / 2, \gamma_{2}=1 / 2$ like [10]

Besides, according to $\mathrm{QE}$ scheme, the Itô integral $\int_{t}^{t+\Delta} \sqrt{\hat{V}(u)} d W(u)$ condition on the distributions of $\hat{V}(t)$ and $\int_{t}^{t+\Delta} \hat{V}(u) d u$, is normal distributed random variable with mean zero and variance $\int_{t}^{t+\Delta} \hat{V}(u) d u$.

Hence, the price process of Heston model can be approximated by:

$$
\hat{S}(t+\Delta)=\hat{S}(t) \exp \left(K_{0}+K_{1} \hat{V}(t+\Delta)+K_{2} \hat{V}(t)+\sqrt{K_{3} \hat{V}(t+\Delta)+K_{4} \hat{V}(t)} Z_{2}\right)
$$

where

$$
\begin{aligned}
& K_{0}=\left(r-\frac{\rho \kappa \theta}{\varepsilon}\right) \Delta, \\
& K_{1}=\left(\frac{\kappa \rho}{\varepsilon}-\frac{1}{2}\right) \gamma_{2} \Delta+\frac{\rho}{\varepsilon}, \\
& K_{2}=\left(\frac{\kappa \rho}{\varepsilon}-\frac{1}{2}\right) \gamma_{1} \Delta-\frac{\rho}{\varepsilon}, \\
& K_{3}=\left(1-\rho^{2}\right) \gamma_{2} \Delta, \\
& K_{4}=\left(1-\rho^{2}\right) \gamma_{1} \Delta,
\end{aligned}
$$

and $Z_{2} \sim N(0,1)$ is independent of $\hat{V}(t)$.

Note that $K_{i}, i=0, \cdots, 4$, depends on the time-step $\Delta$ as well as the constants $\gamma_{1}$ and $\gamma_{2}$.

\section{ES-QE Algorithm} follows:

The algorithm of ES-QE scheme can be summarized as

Step 1: Generate uniform random numbers $U_{1}$ and $U_{2}$, set $Z_{1}=\Phi^{-1}\left(U_{1}\right), \quad Z_{2}=\Phi^{-1}\left(U_{2}\right)$ (for details, to see [14] );

Step 2: Compute the non-central parameter $\lambda$, and then generate Poisson distributed random variable $N$ ( here $N \sim P((1 / 2) \lambda)$ );

Step 3: Given $\hat{V}(t)$, generate $\hat{V}(t+\Delta)$ by Eq.(3);

Step 4: Given $\hat{V}(t), \hat{V}(t+\Delta), \hat{S}(t)$ and $\gamma_{1}, \gamma_{2}$, generate $\hat{S}(t+\Delta)$ by Eq. (4).

\section{NUMERICAL SIMULATION}

To test our discretization scheme, we turn to the numerical pricing of European call options in the Heston model, thus we can computed the analytical result which constitutes a standard test case.
We consider a call option $C$ maturing at time $T$ with strike $K$, the option which underlying price process. For simplicity, we denote $C(0)$ to represent the exact option price at $\tau=0(\tau=T-t), \hat{C}(0)$ is the estimated value of $C(0)$ by ES-QE. We use Monte-Carlo method to get the numerical price of option as follows: First, choose $N$ independent samples from the initial price set of underlying asset $\left\{\hat{S}^{(i)}\left(t_{0}\right)\right\}, i=1,2, \cdots$, Paths ( Paths is the number of simulation paths); and apply our ES-QE scheme to discretize the Heston model equidistantly for $M$ times with time-step $\Delta$, thus yielding Paths independent asset's price set $\left\{\hat{S}^{(i)}\left(t_{j}\right)\right\}$ in which $i=1,2, \cdots$, Paths; $j=1,2, \cdots, M$; then an European call option's price is

$$
\hat{C}(0)=(1 / N) \sum_{i=1}^{N} e^{-r T}\left(\hat{S}^{(i)}(T)-K\right)^{+} .
$$

Due to the bias generated by the discretization of time, $\hat{C}(0)$ is usually not equal to $C(0)$. In general, discretization errors of Monte-Carlo method root in two sources[15] : one is from the discretization simulation process of continuous time, which is known as discretization bias, bias $=|C(0)-\hat{C}(0)|$; the other comes from the estimator's statistic fluctuates of centrallimit theory, known as root of mean-square error(RMS), defined as $R M S=\sqrt{\text { bias }^{2}+\sigma_{f}^{2}}$, where

$$
\sigma_{f}=\operatorname{Std}(C(0)-\hat{C}(0)) / \sqrt{\text { Paths }}
$$

denotes the standard error of samples $\left\{f\left(\hat{S}^{(i)}(T)\right)\right\}$, $i=1,2, \cdots, N$.

To test the accuracy and efficiency of the new scheme, we take the same parameters (listed in Table 1) with BK's in [9] with the spot price 100 and the strike 100 . Two test cases are chosen with respect to the Feller condition. Case 1 is quite easy for many discretization schemes, while Case 2, which is such that the Feller condition is not satisfied, acts as a benchmark test for discretization schemes.

TABLE I. PARAMETERS

\begin{tabular}{ccccccccc}
\hline Case & $\varepsilon$ & $\kappa$ & $\rho$ & $T(\mathbf{y})$ & $V(0)$ & $\theta$ & $r$ & $C(0)$ \\
\hline 1 & 0.61 & 6.21 & -0.70 & 1.0 & 0.010201 & 0.019 & 0.0319 & 6.8061 \\
\hline 2 & 1.00 & 2.00 & -0.30 & 5.0 & 0.09 & 0.09 & 0.05 & 34.9998 \\
\hline
\end{tabular}

All experiments are run with $\Delta=T / 32$. We record the bias and RMS curves of both QE scheme and ES-QE scheme in Figures 1 4. The codes of ES-QE algorithm are written with matlab 2012b, and implemented on a notebook PC with Intel Core i3@2. 20GHz processor and 4G of RAM, running Windows 7 Ultimate. 


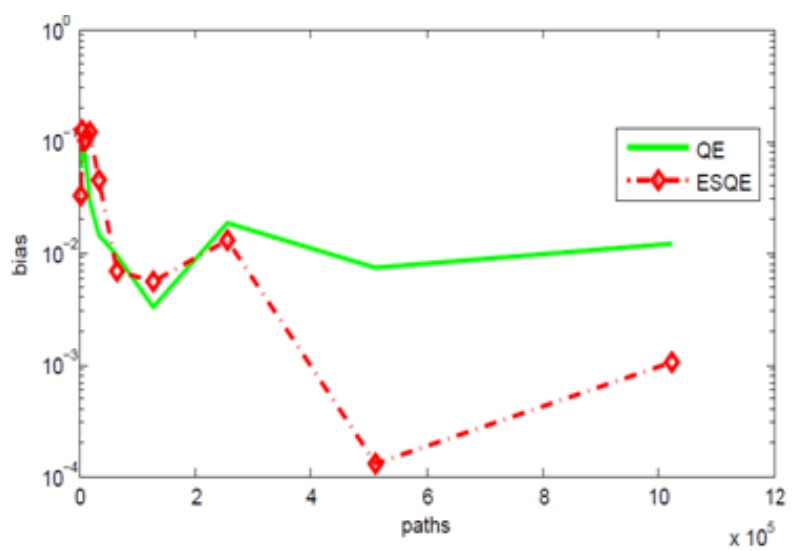

(a) The bias

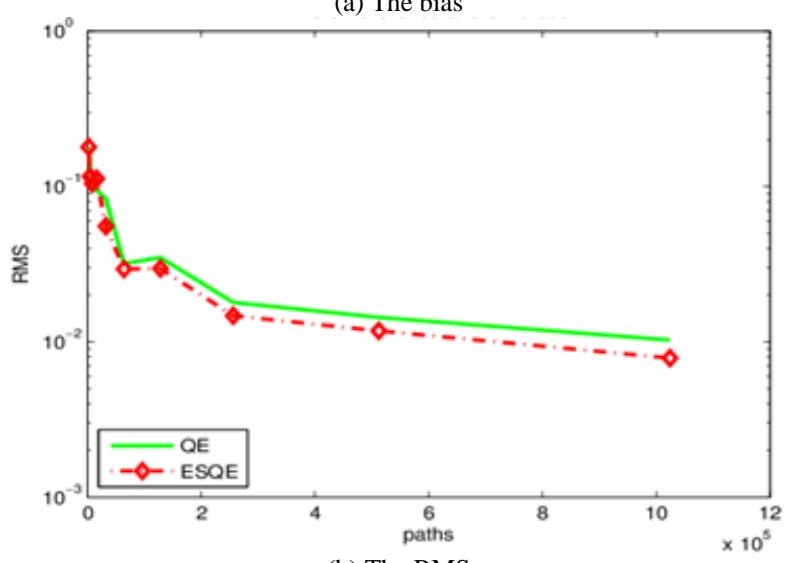

(b) The RMS

FIGURE I. THE ABSOLUTE BIAS AND RMS OF SIMULATION FOR CASE 1

Figure1 (a) and (b) show clearly that as the simulation paths increases, both the absolute bias and RMS of both schemes generally decrease. However, Figure 1 presents that the accuracy of $\mathrm{QE}$ discretization scheme only reaches $O\left(10^{-2}\right)$ even after the Paths comes to the maximum, while our ES-QE gets $O\left(10^{-3}\right)$. Since the RMS reveals not only the bias between analytical solution and estimated value, but also the standard error which reflects the degree of discretization of samples, RMS can serve as a good measure of the accuracy for discretization schemes. Figure 2 shows that ES-QE scheme has higher accuracy than QE scheme.

For the difficult Case 2, Figure 2 (a) and (b) show that the absolute bias and RMS of both schemes decrease gradually as the simulation paths increases, and ES-QE has higher accuracy than QE. All of the results indicate that our ES-QE scheme performs better in the accuracy than QE.
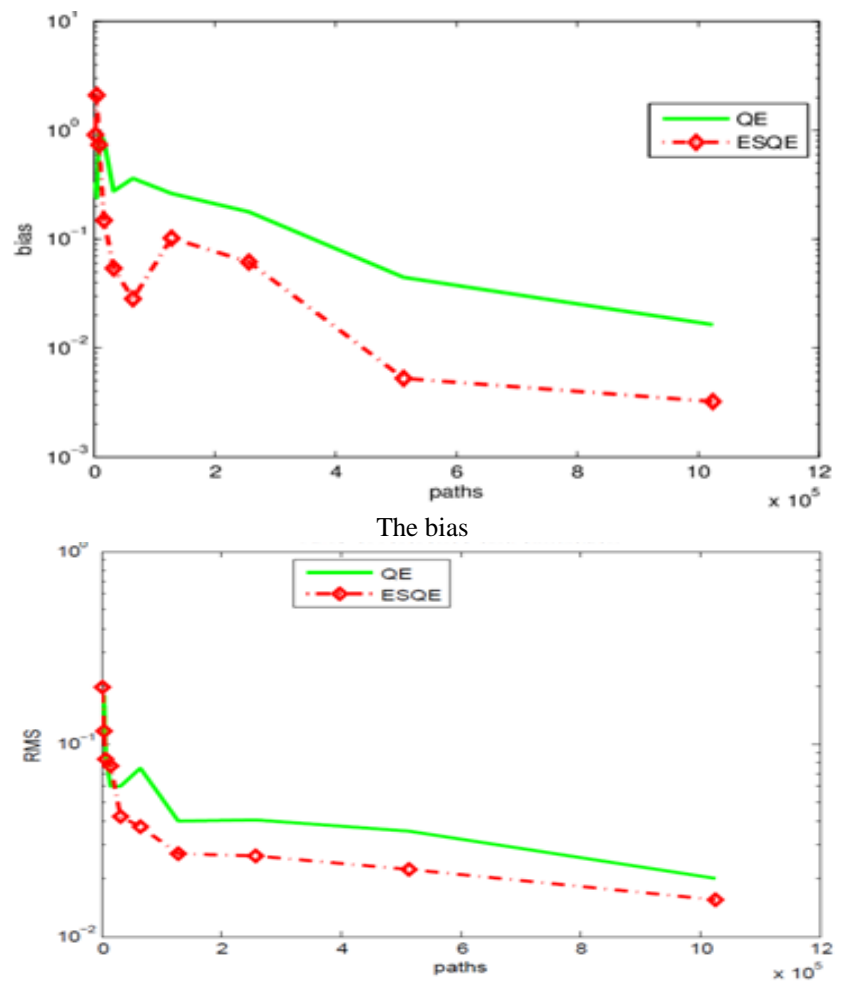

(b) The RMS

FIGURE II. THE ABSOLUTE BIAS AND RMS OF SIMULATION FOR CASE 2

TABLE II. RUN TIME OF DIFFERENT PATHS IN TEST CASE1OF QE AND ES-QE SCHEME

\begin{tabular}{ccccccc}
\hline Paths & 2000 & 4000 & 16000 & 64000 & 256000 & 1024000 \\
\hline QE & $0.116 \mathrm{~s}$ & $0.173 \mathrm{~s}$ & $0.754 \mathrm{~s}$ & $3.328 \mathrm{~s}$ & $9.583 \mathrm{~s}$ & $32.030 \mathrm{~s}$ \\
\hline ES-QE & $0.197 \mathrm{~s}$ & $0.276 \mathrm{~s}$ & $1.132 \mathrm{~s}$ & $4.986 \mathrm{~s}$ & $14.163 \mathrm{~s}$ & $47.272 \mathrm{~s}$ \\
\hline
\end{tabular}

Besides, Table 2 shows the computation efficiency of our scheme. The run time of ES-QE scheme is larger than QE scheme's. However, the computation efficiency of ES-QE is acceptable if compared with that of exact simulation scheme where the run time is measured by hours on the same notebook PC.

\section{CONCLUSIONS}

In this paper, we propose the ES-QE scheme by combining the quadratic exponential (QE) and exact simulation (ES) scheme and take European call option as a benchmark to test the performance of ES-QE. The numerical results show:1) ES$\mathrm{QE}$ scheme has higher accuracy than QE scheme; 2) the run time of ES-QE scheme is larger than QE scheme, but much smaller than ES; 3) both the discretization bias and RMS of ES-QE scheme are less than QE scheme under the same conditions whether the parameters satisfied Feller condition or not . These results indicate that the ES-QE scheme is both effective and feasible.

\section{REFERENCES}

[1] S. Heston. A closed-form solution for options with stochastic volatility with applications to bond and currency options. Review of Financial Studies, 6(2): 327-343, 1993. 
[2] F. Black, M. Scholes. The pricing of options and corporate liabilities . Journal of Political Economy, 81(3):637-654, 1973.

[3] R. Cont. Empirical properties of asset returns: stylize facts and statistical issues. Quantitative Finance, 1(2):223-236, 2001.

[4] A. Haastrecht, A. Pelsser. Efficient, almost exact simulation of the Heston stochastic volatility model. International Journal of Theoretical and Applied Finance, 13(1):1-44, 2010.

[5] W. S. Wadman. An advanced Monte Carlo method for the multi-asset Heston model . Amsterdam: Delft university of technology, 2010.

[6] C. Kahl, P. Jaeckel. Fast strong approximation Monte-carlo schemes for stochastic volatility models. Quantitative Finance, 6(6):513-536, 2006.

[7] R. Lord, R. Koekkoek, D. Dijk. A comparison of biased simulation schemes for stochastic volatility models, Quantitative Finance, 10(2): 177-194, 2010.

[8] J. W. Zhu. A Simple and Accurate Simulation Approach to the Heston Model. The Journal of Derivatives, 18(4): 26-36, 2011.

[9] M. Broadie, O. Kaya. Exact simulation of stochastic volatility and other affine jump diffusion processes . Operations research, 54(2):217-231, 2006.

[10] L. Andersen. Simple and Efficient Simulation of the Heston Stochastic Volatility Model, Journal of Computational Finance, 11(3): 1-42, 2008.

[11] Cox J, Ingersoll J, Ross S. A theory of the term structure of interest rates. Econometrica, 53(2):385-407, 1985.

[12] Johnson, N. L. , Kotz, S. , Balakrishnan. N. Continuous univariate distributions. John Wiley, New York:1994.

[13] D. Dufresne. The integrated square-root process. Working paper, University of Montreal, 2001.

[14] Moro, B. The full Monte. Risk Magazine, 1995, 8(2):57-58.

[15] P. Glasserman, K. Kim, Gamma expansion of the Heston stochastic volatility model, Finance and Stochastic, 15(2): 267-296, 2011.

[16] D. Duffie, P. Glynn. Efficient Monte-Carlo simulation of security prices. The Annals of Applied Probability, 4(4):897-905, 1995. 